\title{
High quality triangular grid generation for the risk analysis of a special lagoon
}

\author{
B. Tansel \\ Istanbul Technical University, Maritime Faculty, Turkey
}

\begin{abstract}
Much of the world's open coasts are formed by estuaries and lagoons which are highly important for ecological reasons. Since the rich organic materials are carried in by the stream water, a favorable environment gets formed for plankton, the other creatures thriving on planktons and the fish feeding on them. On the other hand, the water, the reeds and other vegetation, the fish and the organic material that has settled provide the birds with an ideal environment. In this context, the problems with pollution in lagoon systems are important risk factors for the ecological balance. The purpose of the study is to generate a high quality computational network for the risk analysis of a special lagoon in order to construct a solid foundation for the next step in our study. A reduction of the number of elements is aimed at while the underlying physical domain is adequately represented via several grid refinement criteria. Furthermore, standardizing the method to be used as the calculation network, and utilizing its highly accurate risk predictions for all lagoon systems are also aimed at.
\end{abstract}

Keywords: lagoon quality, risk analyses, grid generation.

\section{Introduction}

Istanbul, one of the largest and crowded metropolitan cities of Turkey, affects coastal fields negatively in terms of city development, as is the case in many examples throughout the world. We performed our study for the Tuzla Kamil Abdus Lagoon (TKAL) in the Tuzla District of Istanbul. Although the fishery and the hosted birds were important ecological elements and also sources of income before the 1970's in the region, it was completely dried out in 2001 as a result of the relocation of the shipyards in this region (which blocked the natural opening of the lagoon), the highway construction, the modified paths of rivers, 
and various other reasons after 1978. The lagoon, which is extremely important in the environmental ecology and from the socio-economical aspects for Tuzla, was rehabilitated and resuscitated by the Municipality of Tuzla and converted into a beneficial facility. Some of the studies on predicting the necessary risks, which are required for the healthy management of TKAL, were summarized in this paper. The purpose of the study related to TKAL is the determination of the security risks, the dimension of these risks and the cases where taking a precaution is necessary in the lagoon. The results of the performed study are not specific to TKAL, but they can be used for all ecologically important lagoons. In our paper, studies on the generation of a mesh structure, which is the basic and the most important phase in the application of this method, was presented. The first phase of the necessary study was completed by obtaining the bathymetry information of the lagoon, and the data on the wind properties and the current data on the region. The second and the most important stage was to construct the mesh structure of the lagoon, which constitutes a basis for the whole analysis. Our next study will be the computerized solution of the equations (the NavierStokes equation and its derivations), by making use of digital methods defining water mass-movements in computer-based models, and following this, the calculation of the fluid free water surface movements and the flow areas will be our next goal. The construction of TKAL mesh structure, which constitutes an important part of this study and the basic results of the first test-run will be presented in our paper.

\section{The triangular grid generation method}

As is known, the coastal areas constitute a rather difficult setup in comparison to a rigid body meshing, due their very complex geometries. Particularly, in order to perform the risk analysis with various wind and current configurations, it is very important to mesh these regions in a correct and meaningful manner. In our study the high quality triangular mesh generation program BatTri was used, which is a public domain meshing program working under Matlab and developed to meet the needs of the ocean modeling researchers [1].

The actual grid generation is performed by Triangle while BatTri does pre and post processing. The Software's stronger part is its Delaunay refinement algorithm (DRAs) during its triangular grid generation. DRA is a method that is used to produce the refined Delaunay triangulation in point sets [2]. According to the Delaunay feature, there are peripheral circles for every triangle, and the triangles are refined in their peripheral circles with certain restrictions. Indeed, BatTri manages the input and output functions with Triangle [1], which performs meshing according to optimizing the conditions on element creation. That is to say, for grid generation and optimization of produced grids, the main program between input and output is the Triangle, which performs re-meshing according to BatTri conditions. In Figure 1 a very general algorithm is shown related to the BatTri meshing loop. 


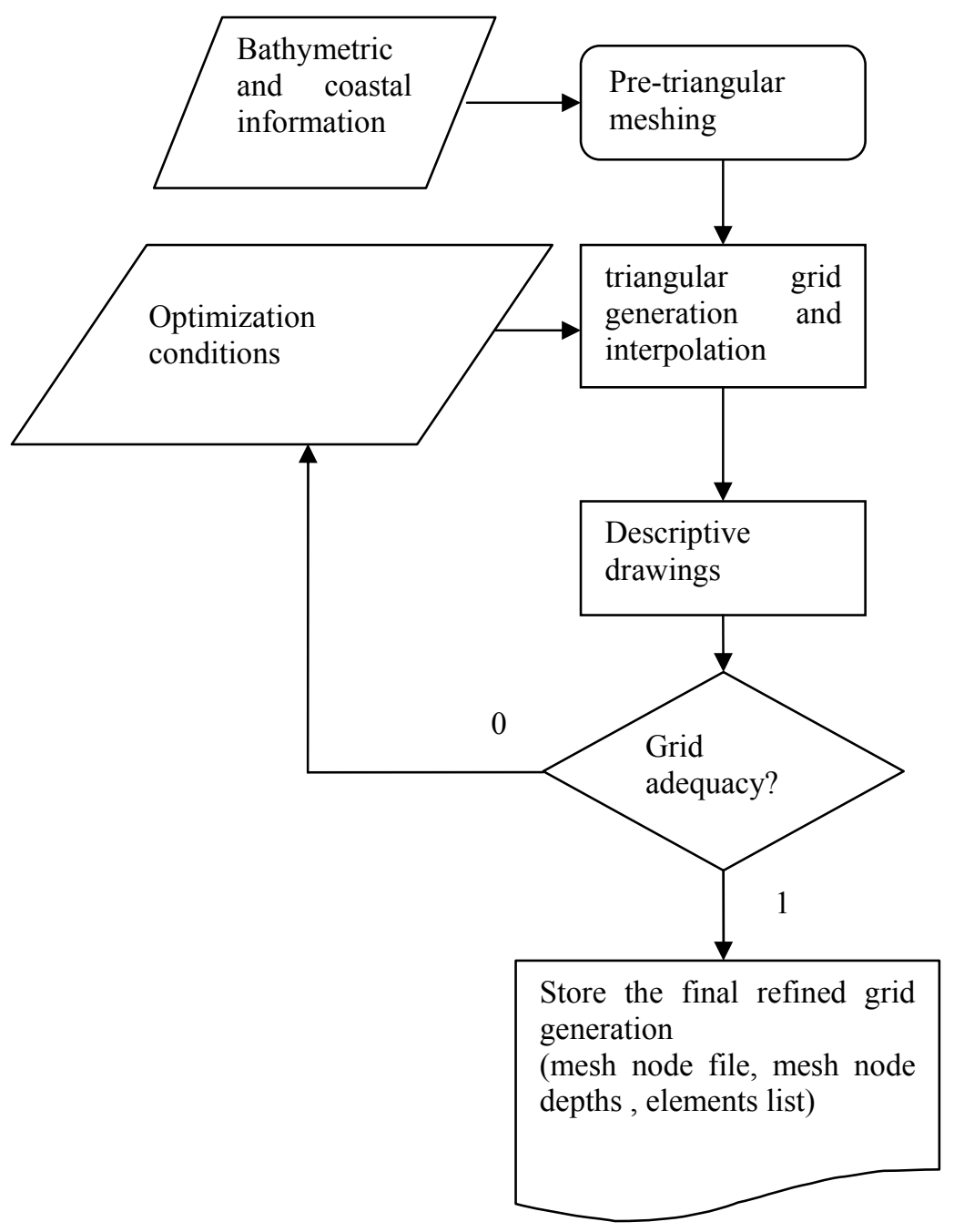

Figure 1: The BatTri Algorithm.

There are BatTri mesh optimization functions for refined grids. Some of these functions are defined below.

$$
\frac{h}{\nabla h} \frac{1}{\alpha} \geq A
$$

$\mathrm{h}$; average element depth, $\boldsymbol{\nabla} h$; depth gradient value of element verticals, A; maximum element area, $\alpha$;limiting ratio stated by the user.

$$
\frac{h}{\alpha} \geq A
$$


The refinement condition related to the tide height and the grid dimension in as of the Courant-Friedrichs-Levy (CFL) type; and for the maximum element area $(\mathrm{A})$,

$$
\frac{g h t^{2}}{R^{2}} \geq A \text { or } \frac{\sqrt{3}}{4} \frac{g h t^{2}}{R^{2}} \geq A
$$

where $\mathrm{g} ; \mathrm{m} / \mathrm{sn}^{2}$ gravity dimension, t; time step of the model in minutes, $\mathrm{R}$; it's the ration tide height of the ebb and the flow to the grid dimension.

The refinement condition as in the Pecklet type; if the current speed and diffusion area are known, this condition can be applied for the restriction of the element area restriction.

$$
\left(\frac{P e D}{V}\right)^{2} \geq A \text { or } \frac{\sqrt{3}}{4}\left(\frac{P e D}{V}\right)^{2} \geq A
$$

where $P e$; Peclet number, $D$; diffusion constant, $V$; speed.

All of these optimization conditions are the functions chosen selected for converting the triangular element into a more refined form [1].

\section{TKAL grid generation studies}

Figure 2 shows the TKAL configuration. As is seen, there are three islands and channels inside the lagoon. The connection of the lagoon with the sea is provided by two openings.

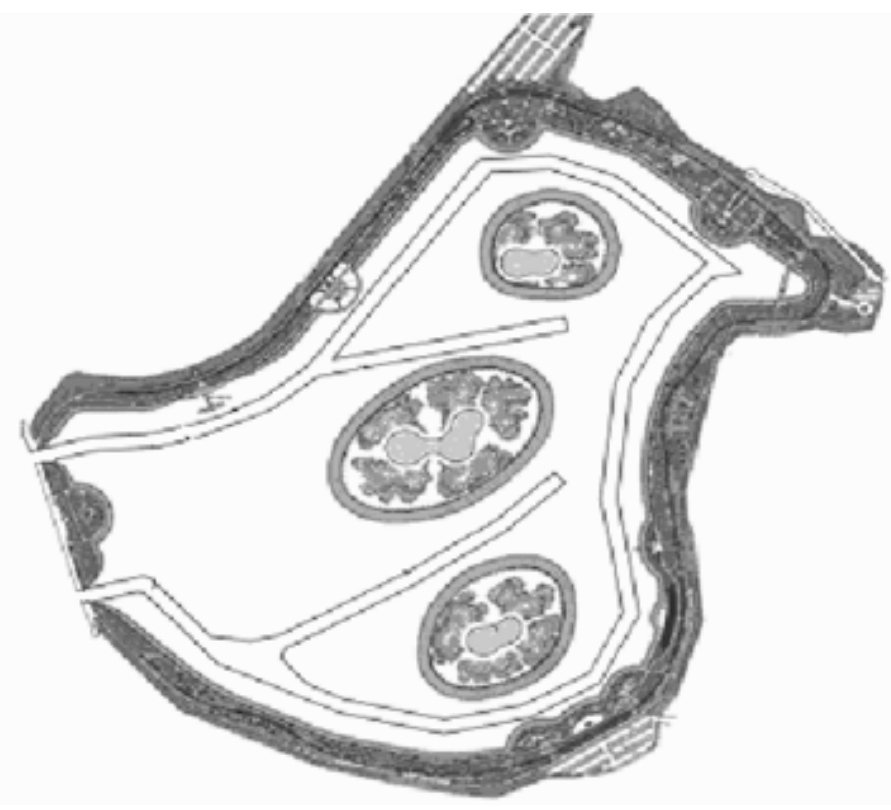

Figure 2: The TKAL configuration. 
The deeper channels were set to be $2.5 \mathrm{~m}$ deeper than the mean sea level and the shallows surrounding the channels were configured to be $1.2 \mathrm{~m}$. Openings were configured to be $20 \mathrm{~m}$ wide and $3.5 \mathrm{~m}$ deep. The transition zone between the inner lagoon channels and the shallows is defined to be $10 \mathrm{~m}$ wide. The depths in this area were randomly selected around a straight line connecting the endpoints of the transition zone with a slope of 0.8 and an average depth of 1.85 $\mathrm{m}$, within a standard variation of $0.492 \mathrm{~m}$. The depths of the intermediate grid points were calculated using linear interpolation. Considering the small tidal range on the order of centimeters $(\approx 6 \mathrm{~cm})$ [3], the absence of fresh water input and the shallowness of the lagoon, it can be assumed that the dominant forces behind the particle exchange with the coastal sea will be caused by the wind stress and the wave action. The dominant winds in the region are southwesterly and northeasterly. In order to effectively resolve the current field in the lagoon and around the lagoon openings where the exchange will occur, two grid resolution zones are defined using the $5 \mathrm{~m}$ isobath. The landward zone contains triangular elements whose areas are always less than $1,000 \mathrm{~m}^{2}$. The open-sea zone contains elements whose areas are less than $10,000 \mathrm{~m}^{2}$. The first base of the analysis of risk examination is created with the high quality meshing via BatTri. On top of this, the deeper channels in the lagoon were marked by nodes that were $5 \mathrm{~m}$ apart along the $2.5 \mathrm{~m}$ isobath. These nodes whose presence is enforced

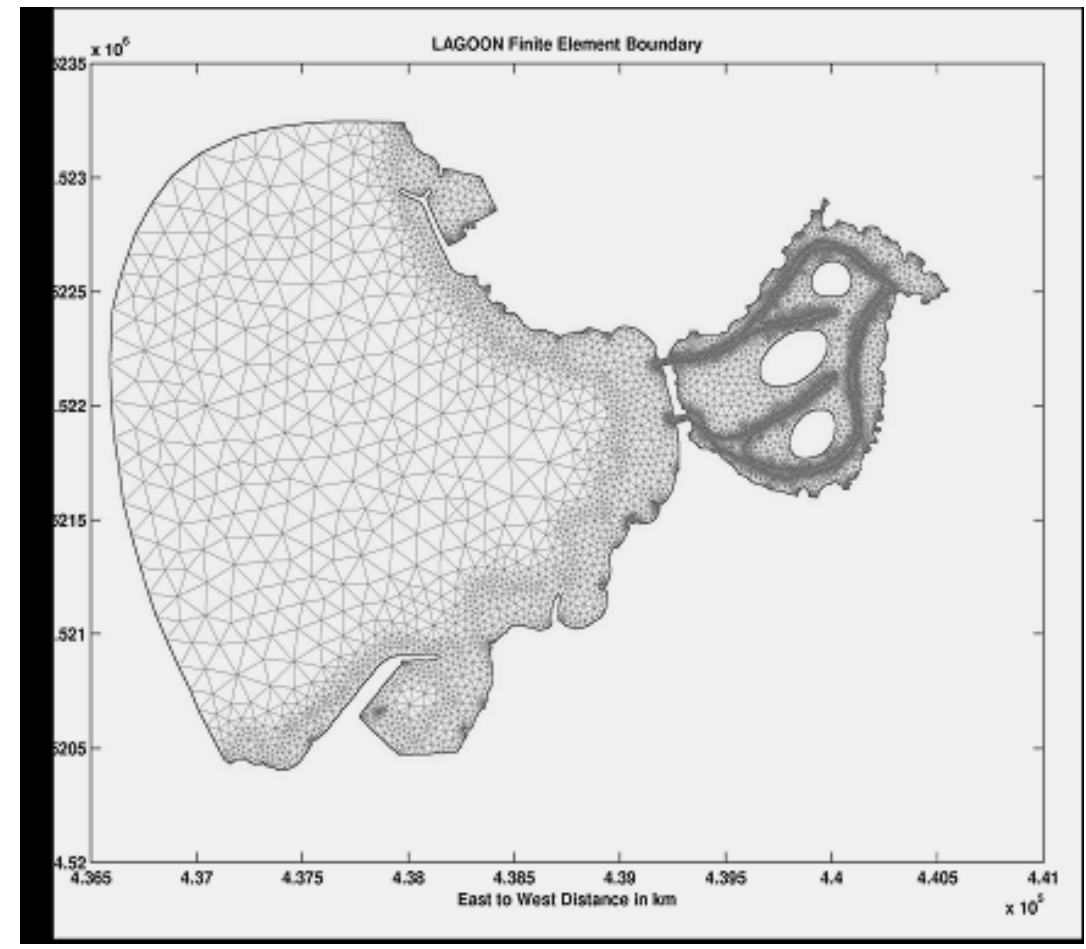

Figure 3: $\quad$ The overall view of the model domain grid. 
in the triangulation assure that the transition zone between the channels and the flats is appropriately resolved by a larger number of elements to simulate the relatively steeper gradients. Also, the larger elements in the open-sea region make sure that the forcing signals are appropriately transferred to the coastal zone and the lagoon, while keeping the number of elements low for obvious computational reasons. The finite element mesh is shown in Figure 3. Bathymetry is shown in Figure 4. The grid consists of 15,347 nodes and 29,287 triangular elements.
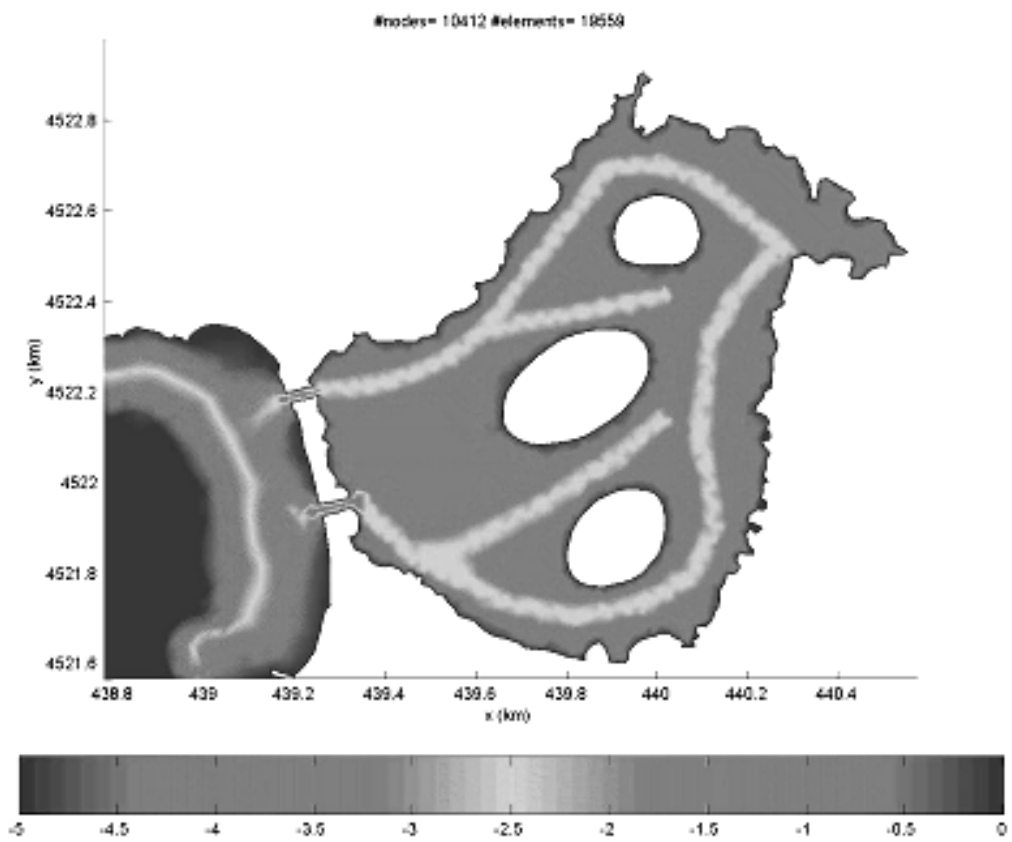

Figure 4: Bathymetric map of the inside and the outside of the lagoon. Scale shows depth in meters.

Figure 5 shows that the element quality is enforced by a minimum $30^{\circ}$ inside angle constraint. This condition is met for a majority of the elements in the domain, except for the regions where the presence of certain nodes are enforced. In these regions, the minimum inside angle may rarely drop down to $25^{\circ}$.

Another requirement that the mesh should meet is the CFL (CourantFriedrichs-Levy) condition, which directly affects the stability of the calculations in the explicit formulations. Assuming equilateral triangles, this can be written as

$$
\frac{\sqrt{3}}{4} \cdot \frac{g \cdot h \cdot t^{2}}{R^{2}}>A
$$

Here, $g$ is the gravitational acceleration, $h$ is the depth of the water, $t$ is the time step, $A$ is the element area and $R$ is a constant. The values of $R$ may range 
between 25 on flat bottoms and 500 on high bathymetry gradients. $R=100$ would be an optimized choice for many situations. Assuming that the maximum current velocities in the TKAL model domain are in the vicinity of $0.5 \mathrm{~m} / \mathrm{sec}$, the time steps on the order of 25 seconds may be needed to meet the CFL condition. Figure 6 shows the time steps.

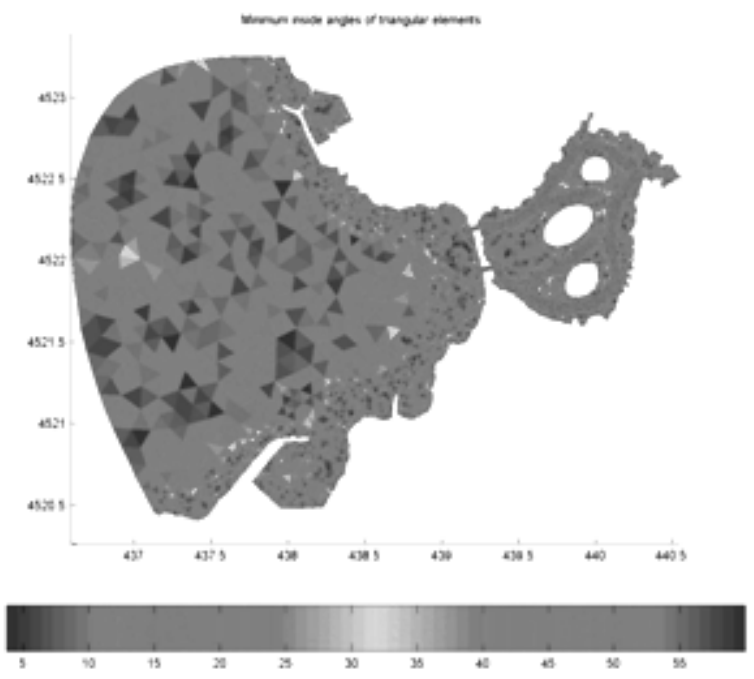

Figure 5: The representation of the Lagoon inner mesh structure based on the inner angles, refined according to the BatTri program.

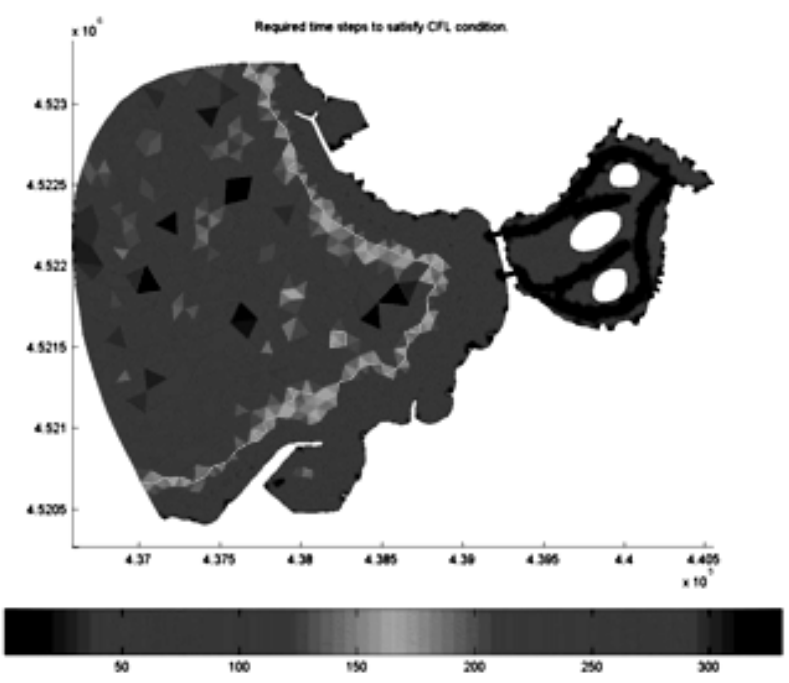

Figure 6: The regional time steps determined according to the BatTri. 
Figure 7 shows the element quality measure as defined by:

$$
q=\frac{4 \cdot \sqrt{3} \cdot A}{L_{1}{ }^{2}+L_{2}{ }^{2}+L_{3}{ }^{2}}
$$

Here, $q$ is the element quality measure, $A$ is the element area and $L_{1}, L_{2}, L_{3}$ are the element edge lengths. It is usually assumed that the element quality is acceptable for all $\mathrm{q} \geq 0.6$. Please note that in the ideal situation where an element is an equilateral triangle, $q=1$ since, $L_{1}=L_{2}=L_{3}$.

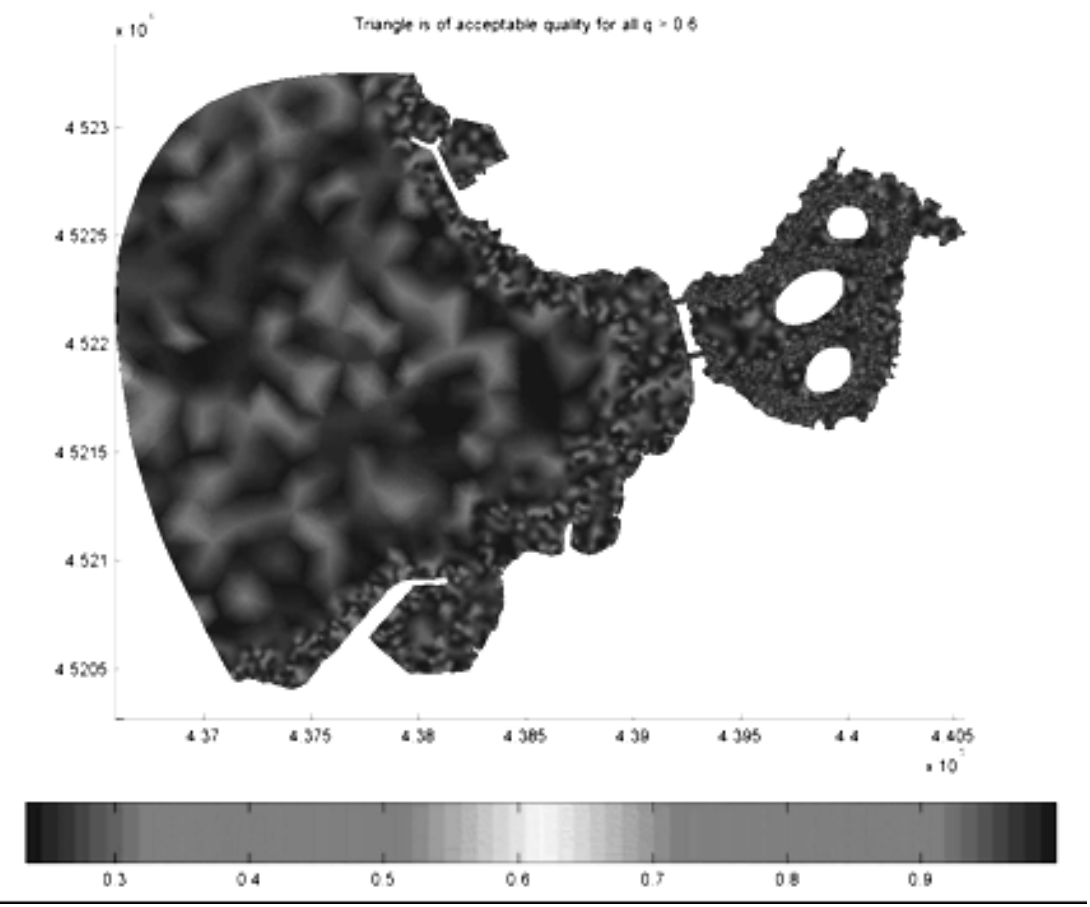

Figure 7: The quality map of the calculation mesh elements. The scale shows the element quality measure $q$. The dark part, represents $q=1$.

The characteristic element length of the grid, as calculated by $\sqrt{2 \cdot A}$, where $A$ is the element area, ranges from $0.10 \mathrm{~m}$ in the lagoon to $153 \mathrm{~m}$ in the open-sea, with an average of $11.17 \mathrm{~m}$ and a standard variation of $16.9 \mathrm{~m}$.

A model run with the standardized input performed on the generated grid showed no overall qualitative problems in the calculated current fields. For the quantitative evaluations, a field work is planned to acquire the current profiles in and out of the lagoon for the summer of 2010. Two examples of the resulting current vector fields are shown in Figures 8 and 9. The findings demonstrated in Figures 8 and 9 were obtained with the calculation network standard inputs by 


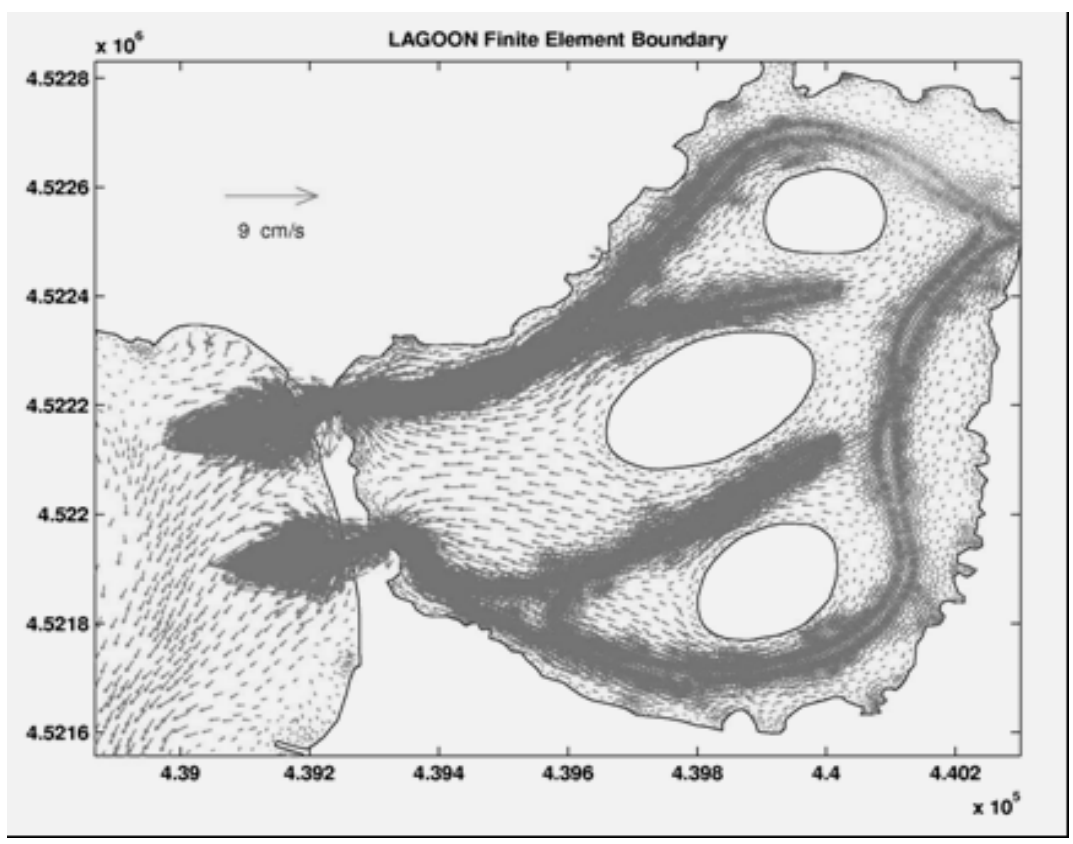

Figure 8: Characteristic example flow vectors obtained as a result of test run around the lagoon.

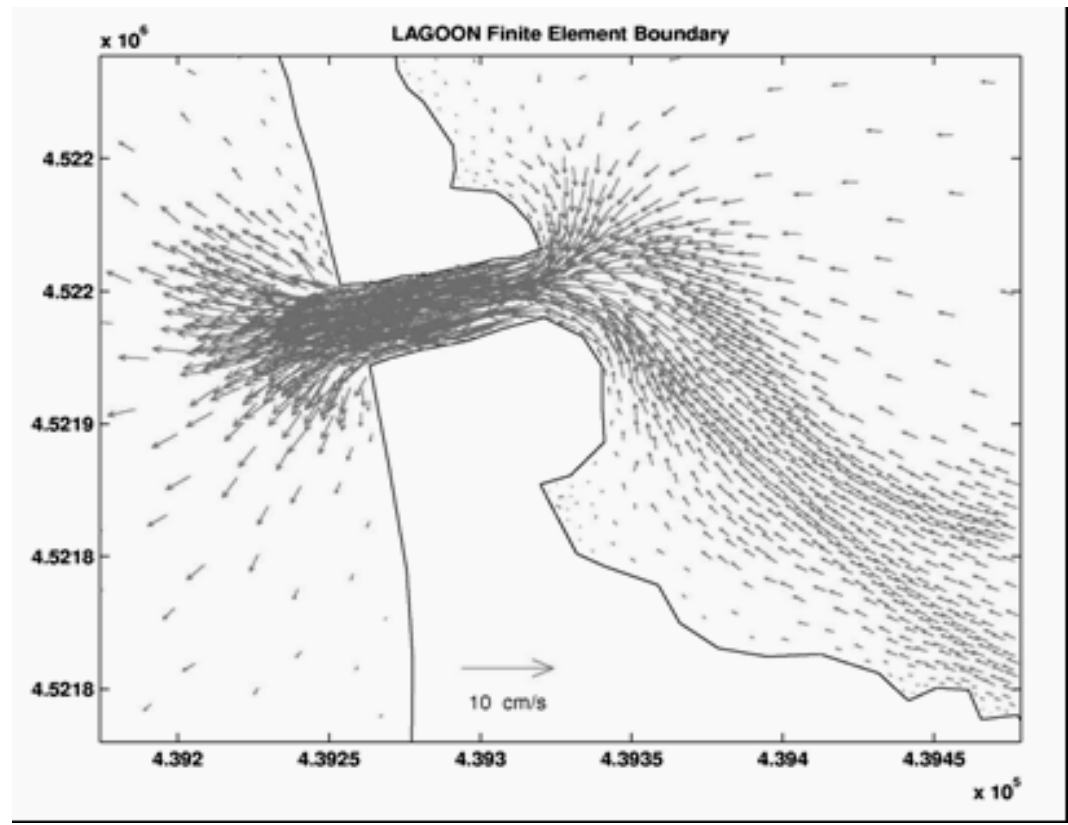

Figure 9: The characteristic example flow vectors obtained as a result of the test run for the southern lagoon opening. 
running in the Cygwin environment using a laptop computer with $3.20 \mathrm{MHz}$ and 1 GB RAM. A simulation of 24 hours was completed in 51 minutes with a time step of 144 seconds. In conclusion, no anomaly connected with the calculation network has been observed in the analysis of the calculated velocity vectors. The network transfers the signals that belong to the open-sea free-water level and wind pressure to the coastal area and the lagoon, and vectors in these areas react to these actions. In the phase of real-time modelling, it is estimated that no problem in terms of the calculation network will be experienced in order to ensure the statistical validity of the findings to be obtained through the optimization of input parameters. The current vector maps of test conditions were only provided as characteristic examples.

\section{Conclusion}

A base grid on which the hydrodynamic finite element simulations are going to be performed has crucial importance on the quality of results. Several factors such as the choice of the model time steps (via CFL condition) and the dispersion coefficients (via Peclet number) actually depend on the characteristics of the grid, combined with the hydrodynamic vector field properties. The optimization here is of utmost importance: an excessive number of elements should be eliminated while the domain discretization is ensured adequately. In this study, a high quality finite element grid is generated for the Tuzla Kamil Abdus Lagoon in Istanbul, Turkey using the public domain triangular finite element grid generator BatTri. Several important parameters such as the quality of the triangular elements and the model time step were examined and determined for later simulations. A test simulation performed on the grid showed no qualitative problems.

Simulations of the lagoon water quality using statistically proven computer models is much cheaper than making a number of measurements, and has the benefit of examining effects of different input parameters in a quick, easy and reliable way. The fast generation of a reliable grid will help in not only normal cases but also in emergency situations for quick decision making.

\section{References}

[1] Bilgili, A. \& Smith, K.W., BatTri: A two-dimensional bathymetry-based unstructured triangular grid generator for finite element circulation modeling. Lynch Computers and Geosciences 32:632-642. 2006.

[2] Lee, D.T. \& Schachter, B.J., Two algorithms for constructing a Delaunay triangulation. International Journal of Computer Information Science 9, 219-242. 1980.

[3] Alpar, B. \&, Yüce H., Sea-level Variations and their Interactions between the Black Sea and the Aegean Sea. Estuarine, Coastal and Shelf Science 46, 609-619, 1998. 